\title{
An approach via fuzzy systems for dynamics and control of the soybean aphid
}

\author{
Magda S. Peixoto ${ }^{1}$ Laécio C. Barros ${ }^{2}$ Rodney C. Bassanezi ${ }^{3}$ Odair A. Fernandes ${ }^{4}$ \\ ${ }^{1}$ DFQM, Universidade Federal de São Carlos (UFSCar), Sorocaba, São Paulo, 18052-780, Brazil \\ ${ }^{2,3}$ DMA, Universidade Estadual de Campinas (UNICAMP), Campinas, São Paulo, 13083-859, Brazil \\ ${ }^{4}$ FCAV, Universidade Estadual Paulista (UNESP), Jaboticabal, São Paulo, 14884-900, Brazil
}

\begin{abstract}
This paper uses a methodology based in fuzzy sets theory in order to describe the interaction between the prey, Aphis glycines (Hemiptera: Aphididae) the soybean aphid, and its predator, Orius insidiosus (Hemiptera: Anthocoridae) and to propose a chemical control for soybean aphid. Economic thresholds were already developed for this pest. The model includes biotic (predator) and abiotic (temperature) factors, which affect the soybean aphid population dynamics. This model is very useful to predict timing and releasing numbers of predators for soybean aphid biological control.
\end{abstract}

Keywords: systems; Mamdani inference method; prey; predator; Aphis glycines; control.

\section{Introduction}

The soybean aphid, Aphis glycines (Hemiptera: Aphididae), is an invasive herbivore new to North America. It was first discovered in North America in Wisconsin in late July 2000 infesting soybean crop. Natural enemies have been observed to attack this pest. An economic threshold was developed for chemical control, i.e. when an insecticide treatment is warranted. Economic thresholds for the soybean aphid have been developed and vary from 250 to 273 aphids per plant [8].

This work describes a method based in Mamdani Fuzzy Control [6] to build a model to evaluate the interaction of the prey, A. glycines (the soybean aphid) and the predator, O. insidiosus (Hemiptera: Anthocoridae), considering the effects of the temperature in the growth of the prey population [10]. Subsequently, we propose a fuzzy chemical control to soybean aphid.

The dynamic model results in a fuzzy model that preserves the biological meaning and nature of the predator-prey model, that is, the fluctuations in the population density of both species and the peaks in the density of the prey and predator alternate. The aim of this investigation is to propose a specific method to enhance current decision making tools to control this pest. The soybean aphid is still a quarantine pest in Brazil. Therefore, before any eventual invasion, a predictive model to enhance biological control program is desirable, because the predator is present in Brazil.

Brazil is the second largest exporter of soybean at present, after the USA and before Argentina. According to the Bureau of Agriculture of the USA, it has been estimated that Brazil will be the largest soybean exporter in 2023. Therefore, considering the economic importance of soybeans in Brazil, we need to be prepared for effective proposals to control soybean aphids or any other invasive pest.

\section{Soybean aphid}

The soybean aphid is a small sap-succking and partenogenetically reproducing insect. Females are produced during spring and summer. During lte summer or early fall, the population will produce winged males along special winged females called gynoparae. These aphids move to their overwintering host, buckthorn, Rhamnus sp., where mating occurs and at least one generation occurs. It is one of the major insect pests of soybean reproducing stages when high populations may build up and can cause great damage [7].

Economic thresholds for the soybean aphid have been developed and vary from 250 to 273 aphids per plant [8]. These thresholds are mainly for pesticide use, despite the fact that some naturally occurring predators such as the multicolored Asian lady beetle, Harmonia axyridis (Coleoptera: Coccinelidae) and the insidious flower bug, Orius insidiosus (Hemiptera: Anthocoridae), the most common and important predator [8]. The insidious flower bug can significantly slow soybean aphid population growth, particularly during hot summer weather [9].

Soybean aphid populations can grow to extremely high levels under favorable environmental conditions. Reproduction and development is faster when temperatures are between $25^{\circ} \mathrm{C}$ and the mid $29^{\circ} \mathrm{C}$ when population can double in two or three days. The aphids are greatly affected by temperatures above $30^{\circ} \mathrm{C}$, and they are reported to begin to die when temperatures reach $35^{\circ} \mathrm{C}[10]$.

\section{Predation}

Predation is an example of the interaction between two populations that results in negative effects on growth and survival of one population (prey) and 
in positive or beneficial effects to the other (predator). A predator is an organism that hunts and kills other organisms for food [1]. Prey populations grow when predators are absent. Predators depend on the presence of their prey to survive. An encounter is assumed to decrease the prey population and increase the predator population by contributing to their growth.

We present hypotheses that characterize a predator-prey model, that is:

- if the number of predators is small, the number of prey increases;

- if the number of predators is large, the number of prey decreases;

- if the number of prey is large, the number of predators increases;

- if the number of prey is small, the number of predators decreases.

According to these hypotheses, we have established fuzzy rule base instead of usual differential equations that characterize the classic deterministic models. This rule base replaces differential equations, which characterize the classic deterministic models used to model the dynamics between prey and predators. These hypotheses are qualitative information that allow us to propose rules that relate (at least partially), the numbers in the populations with their own variations [11]. In fact, our main interest in this paper is to elaborate a predator-prey model that represents the interaction between soybean aphids (prey) and their predators using fuzzy rule-based systems.

\section{The mathematical model for the interaction between soybean aphids and their predator}

Next we develop brief reviews of the concept of fuzzy set and fuzzy rule-based system, and we detail the fuzzy model suggested in this paper.

\subsection{Fuzzy rule-based system}

Fuzzy sets and fuzzy logic have become one of the emerging areas in contemporary technologies of information processing. Fuzzy Sets Theory was first developed by Zadeh [12] in the mid-1960s to represent uncertain and imprecise knowledge. It provides an approximate but effective means of describing the behavior of the system that is too complex, ill defined, or not easily analyzed mathematically, and this is our case.

A fuzzy set $A$ is characterized by a membership function, $\psi_{A}$, mapping the elements of a domain $X$ to the unit interval $[0,1]$. That is, $\psi_{A}: X \rightarrow[0,1]$. Clearly, a fuzzy set is a generation of the concept of a set whose membership function takes on only two values $\{0,1\}$, that is, the characteristic function of $A, \chi_{A}: X \rightarrow\{0,1\}$.
Fuzzy variables are processed using a fuzzy rulebased system. A general fuzzy rule-based system consists of four components: an input processor (fuzzification), a fuzzy rule base; a fuzzy inference method and an output processor (defuzzification). These components process real-valued inputs in order to provide real-valued outputs.

The fuzzification is the process in which the input values of the system are converted into appropriate fuzzy sets of their respective universes. It is a mapping of the domain of real numbers to a fuzzy range [2]. Expert knowledge plays an important role in building the membership functions for each fuzzy set associated with the inputs.

The rule base characterizes the objectives and strategies used by specialists in the area through a linguistic rule set. It is composed of a collection of fuzzy conditional propositions in the form if-then rules $[2]$.

The fuzzy inference method performs an approximate reasoning using the compositional rule of inference. A particular form of fuzzy inference of interest here is Mamdani's method [3]. In this case, it aggregates the rules through the logical operator OR, modeled by the maximum operator and, in each rule, the logical operators AND and THEN are modeled by the minimum operator [3]. The logic of decision to be made, incorporates the structure of inference of the rule base and uses fuzzy implications to simulate the decisions [2]. It generates actions inferred from consequents a set of input conditions - antecedents.

Finally, in defuzzification, the value of the output linguistic variable inferred from the fuzzy rule is translated to a real value. The output processor's task is to provide real-valued outputs using defuzzification which is a process that chooses a real number that is representative of the inferred fuzzy set. A typical defuzzification scheme adopted in this paper is the centroid or center of mass method [3].

According to [2], fuzzy set approaches have been developed for special purposes where the information base is vague and/or imprecise. Under these conditions, fuzzy techniques allow more accurate conclusions in comparison to the other approaches which cannot be applied successfully because of lack of data.

\subsection{The formulation of mathematical model}

Our fuzzy mathematical model is composed of a predator-prey fuzzy system considering the temperature data.

The variables of the fuzzy system are number of prey - $x$-, number of the predators - $y$ - (input variables) and their variations (output variables). The fuzzy sets of the input variables are $\{$ small, small medium, large medium, large $\}$ (that is, the triangular functions defined in the interval $[0 ; 1000]$ for the number of prey and in the interval $[0 ; 0.3]$ for the number of predators) and the fuzzy sets of the 


\begin{tabular}{|c|c|c|c|c|}
\hline Temperature & $20^{\circ} \mathrm{C}$ & $25^{\circ} \mathrm{C}$ & $30^{\circ} \mathrm{C}$ & $35^{\circ} \mathrm{C}$ \\
\hline $\begin{array}{c}\text { Intrinsic rate } \\
\text { of increase }\end{array}$ & 0.368 & 0.474 & 0.375 & -0.383 \\
\hline
\end{tabular}

Table 1: Data of soybean aphids at four constant temperatures on soybean. Source: [10].

output variables are $\{$ small positive, large positive, small negative, large negative $\}$ (that is, the triangular functions defined in the interval $[-0.4 ; 0.4]$ for the variation of the prey $-x /$ - and in the interval $[-0.1 ; 0.2]$ for the variation of the predators $\left.-y^{\prime}\right)[5]$.

Considering the hypotheses of the predation, we have elaborated 16 rules of the system (S1):

1. If ( $x$ is small) and ( $y$ is small) then ( $x /$ is largepositive) and ( $y$ ' is large-negative)

2. If ( $x$ is small-medium) and ( $y$ is small) then ( $x$ ' is large-positive) and ( $y /$ is small-negative)

3. If ( $x$ is large-medium) and ( $y$ is small) then $(x \prime$ is large-positive) and ( $y$ ' is small-positive)

4. If ( $x$ is large) and ( $y$ is small) then ( $x /$ is largepositive) and ( $y$ ' is large-positive)

5. If ( $x$ is small) and ( $y$ is small-medium) then ( $x$ ' is small-positive) and ( $y$ ' is large-negative)

6. If ( $x$ is small-medium) and ( $y$ is small-medium) then ( $x \prime$ is small-positive) and ( $y \prime$ is smallnegative)

7. If ( $x$ is large-medium) and ( $y$ is small-medium) then ( $x \prime$ is small-positive) and ( $y \prime$ is smallpositive)

8. If ( $x$ is large) and ( $y$ is small-medium) then ( $x$ ' is small-positive) and ( $y$ ' is large-positive)

9. If ( $x$ is small) and ( $y$ is large-medium) then ( $x$ ' is small-negative) and ( $y$ / is large-negative)

10. If ( $x$ is small-medium) and ( $y$ is large-medium) then ( $x \prime$ is small-negative) and ( $y^{\prime}$ is smallnegative)

11. If ( $x$ is large-medium) and ( $y$ is large-medium) then ( $x /$ is small-negative) and ( $y \prime$ is smallpositive)

12. If ( $x$ is large) and ( $y$ is large-medium) then ( $x$ ' is small-negative) and ( $y$ / is large-positive)

13. If ( $x$ is small) and ( $y$ is large) then ( $x /$ is largenegative) and ( $y$ ' is large-negative)

14. If ( $x$ is small-medium) and ( $y$ is large) then ( $x$ ' is large-negative) and ( $y$ ' is small-negative)

15. If ( $x$ is large-medium) and ( $y$ is large) then ( $x$ ' is large-negative) and ( $y$ ' is small-positive)

16. If ( $x$ is large) and ( $y$ is large) then ( $x /$ is largenegative) and ( $y /$ is large-positive)

Hence, we have obtained the variation rates of the populations from Mamdani Inference method and the fuzzification of the center-of-gravity.

Regarding the influence of the temperature abiotic factor in the population of soybean aphids, McCornack et al. [10] got the intrinsic rate of increase (1) as:

Using an adjustment from a least-squares method, we have estimated an intrinsic rate of in- crease:

$\Delta x_{t}=0.0006 * T^{3}+0.413 * T^{2}-0.9142 * T+6.9740$

where $T$ is the temperature in instant $t$.

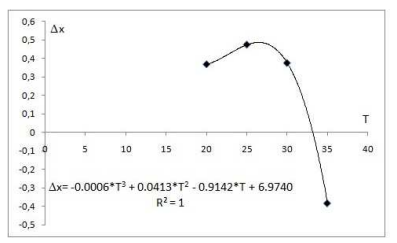

Figure 1: Temperature $(T) \mathrm{x}$ intrinsic rate of increase of soybean aphids $(\Delta x)$.

In the numerical simulations performed we have observed the variation of the number of the prey and the number of the predators considering the temperature. In order to achieve this, we have considered an initial number of aphids, $x_{0}=x\left(t_{0}\right)$, an initial number of predators, $y_{0}=y\left(t_{0}\right)$, in a plant and the temperature, $T_{0}$ in the instant $t_{0}$. From (2) we have obtained $\Delta x_{0}$ (variation of $x_{0}$ due the temperature in $t_{0}$ ). The fuzzy predator-prey system produces $x \prime_{0}$ and $y \prime_{0}$ as output values from the initial conditions and $\Delta x_{0}$ updates $x \prime_{0}$ (that is, $x \prime_{0} \leftarrow x \prime_{0}+\Delta x_{0}$ ) in order to get $x_{1}$ and $y_{1}$. For each iteration, we have the following:

$$
\begin{aligned}
& x\left(t_{i+1}\right)=x\left(t_{i}\right)+h * x \prime\left(t_{i}\right) \\
& y\left(t_{i+1}\right)=y\left(t_{i}\right)+h * y \prime\left(t_{i}\right)
\end{aligned}
$$

that is, Euler's Method, where $h$ is the increment.

\section{Results}

\subsection{Computer simulations}

We have obtained the variation of the number of the prey and the number of the predators in the numerical simulation. Let be $x_{0}=x\left(t_{0}\right)$ an initial number of aphids and $y_{0}=y\left(t_{0}\right)$ be an initial number of predators per plant (input variables of the system (S1)) and the time-at-temperature derived from Figure 2.

Simulations of the trajectories produced by the fuzzy model follow the steps below:

- given an initial number of the prey population $\left(x\left(t_{0}\right)\right)$ and an initial number of the predator population $\left(y\left(t_{0}\right)\right)$ as input data of the one fuzzy rule-based system (S1);

- the fuzzy rule-based system of the predatorprey type gives the output data: $x \prime\left(t_{0}\right)$ and $y \prime\left(t_{0}\right)$

- given an initial temperature $T$ (mean temperatura in Figure 2), by (1) we estimate $\Delta x_{0}$;

- $x \prime_{0} \leftarrow x \prime_{0}+\Delta x_{0}$

- from (2) we find $x\left(t_{1}\right)$ and $y\left(t_{1}\right)$; 

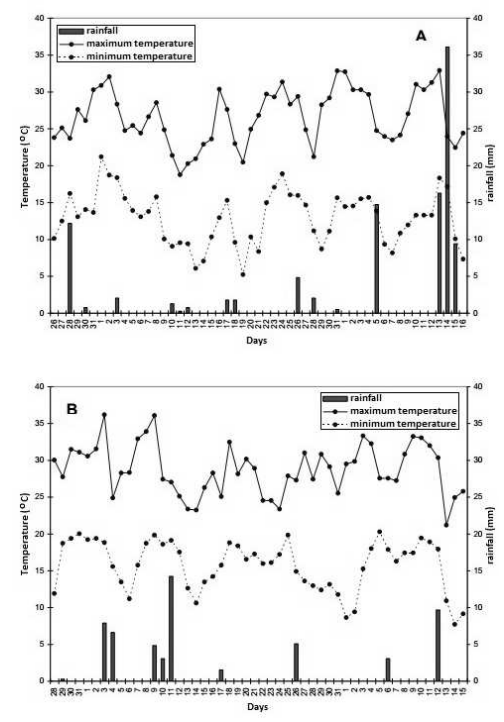

Figure 2: Climatic parameters (temperature and rain) registered during the soybean aphid infestation periods in 2004 (A) and 2005 (B). Data obtained from weather station at Haskell Agricultural Laboratory (Northeast Research and Extension Center), Concord, NE, USA.

- $x\left(t_{1}\right)$ and $y\left(t_{1}\right)$ are the input data of the fuzzy rule-based system of the predator-prey type (S1) and so forth.

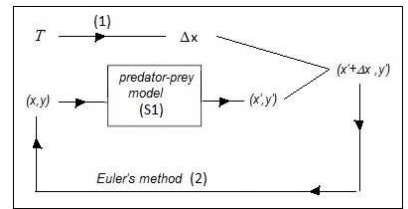

Figure 3: Structure of the mathematical model to obtain the populations $x(t)$ and $y(t)$.

The phase plane by this fuzzy system (dashed line) and the phase plane by the real data [13] (black points) are illustrated in figures 4 and 5 .

\subsection{Discussion}

In section 4.2 we propose the fuzzy model to simulate soybean aphid population dynamics, that includes biotic (predator) and abiotic (temperature) factors. This model is based on fuzzy system (S1) that relate the input variables (number of prey, number of predators) with the output variables (variation of prey and variation of predators) and (1). The use of a fuzzy rule-based system instead of usual differential equations which characterize the classic deterministic models, because many parameters of the differential equations are not available. On the other hand, the qualitative information and data reported in the literature [13] could contribute to the appropriate elaboration of the rule base and the fuzzy sets.

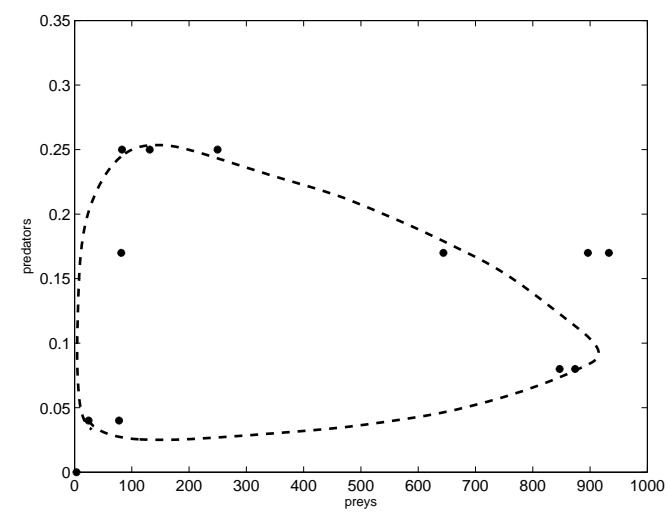

Figure 4: The phase plane by the fuzzy system and the phase plane by the real data with $x_{0}=24.46$ (initial number of aphids), $y_{0}=0.04$ (initial number of predators) and the temperature are available in Figure 2 in 2004.

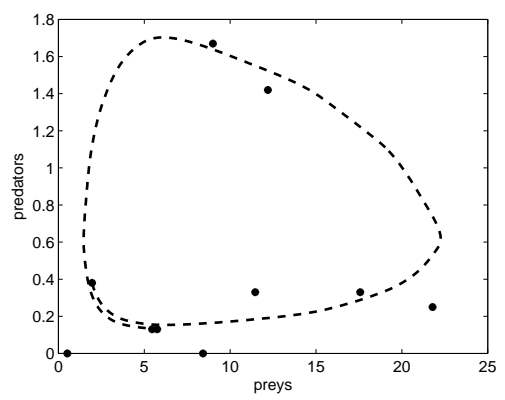

Figure 5: The phase plane by the fuzzy system and the phase plane by the real data with $x_{0}=1.96$ (initial number of aphids), $y_{0}=0.38$ (initial number of predators) and the temperature are available in Figure 2 in 2005.

Section 5.1 demonstrates that the fuzzy mathematical model provides the phase plane that preserves the characteristics of the phase plane of a predator-prey model, that is, dynamic model results in a fuzzy model that preserves the biological meaning and nature of the predator-prey model.

As demonstrated by the simulations in Section 5.1 , we can see clearly that the temperature influences the growth of the aphids population [10] and a comparison between the fuzzy model and real data [13].

We believe that this mathematical model will be very useful to predict the timing and the number of predators released for soybean aphid biological control. Although this pest is not currently present in South America, the model is important to simulate possible scenarios in a soybean plantation.

\section{The fuzzy control}

The soy growers apply insecticide to eliminate this pest. In this case, we have decided to model the 
quantity of the insecticide applied that depends on the number of aphids present in the plants, that is, the larger the density of aphids, the greater the quantity of insecticide. This quantity of the insecticide - the variable $I$ - dependent on the quantity of the soybean aphids present in the plants - the variable $x$ - will be modeled by fuzzy rule-based system. Hence, the variables of this system are the number of prey (input variable) and the quantity of the insecticide (output variable). The fuzzy sets of the input variable are still the same of fuzzy predatorprey system (S1) and the fuzzy sets of the output variable are low, medium, high (that is, the triangular functions defined in the interval $[50,100]$ ).

In this way, we propose the following rule base of the fuzzy control system (S2):

1. If ( $\mathrm{x}$ is small) then ( $\mathrm{I}$ is low)

2. If ( $\mathrm{x}$ is small-medium) then ( $\mathrm{I}$ is low)

3. If ( $\mathrm{x}$ is large-medium) then ( $\mathrm{I}$ is medium)

4. If ( $\mathrm{x}$ is large) then ( $\mathrm{I}$ is high)

We obtain the solution curve from Mamdani Inference Method and the fuzzification of the centerof-gravity represented in Figure 6.

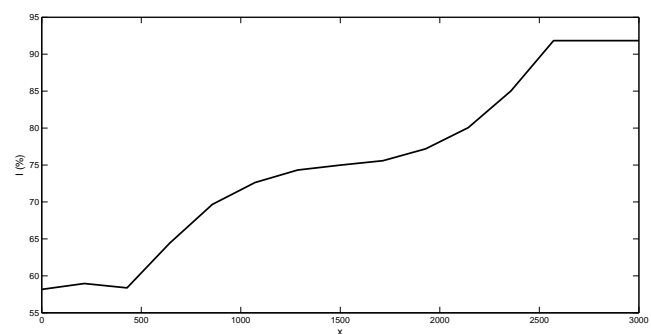

Figure 6: The solution of the fuzzy system: $I=I(x)$.

Figure 6 illustrates the quantity of the insecticide that might be applied depending on the number of soybean aphids, i.e., from the populational density of aphids we obtain the percentage of the population to be removed from the plant by fuzzy control.

We suggest using this as a policy of the control of soybean aphids, because the fuzzy system provides how often and how much to apply the quantity of insecticide on the plants.

\subsection{Computer simulations}

A method to determine if an insecticide treatment is warranted, is a conventional method using the 250 aphids per plant as the economic threshold with $80 \%$ of the plants infested and aphids population increasing [8].

Considering the above-mentioned hypotheses, we have calculated the variation in the number of prey and the number of predators in a numerical simulation. Let be $x_{0}=x\left(t_{0}\right)$ an initial number of soybean aphids (input variable of the fuzzy system) and $y_{0}=y\left(t_{0}\right)$ be an initial number of predators per plant by fuzzy predator-prey system (S1).
From $x_{0}$ we obtain the quantity of insecticide variable, $I$, using the fuzzy system $(\mathrm{S} 2)$. In this way, we obtain the percentage of both populations to be removed from the plant as depicted, that is:

$$
\begin{aligned}
x\left(t_{i+1}\right) & =x\left(t_{i}\right)-I * x\left(t_{i}\right) \\
y\left(t_{i+1}\right) & =y\left(t_{i}\right)-I * y\left(t_{i}\right)
\end{aligned}
$$

in Figure 7.

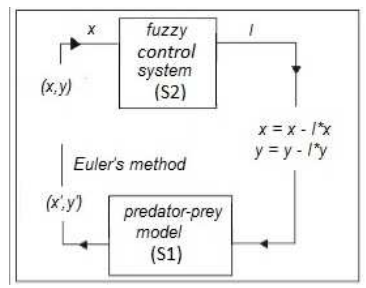

Figure 7: Structure of the fuzzy control.

The evolution of the population is contingent on the prey and predators over time given by the fuzzy model is depicted in figures 8 and 9 with apllication of the insectide, when the population of prey exceed 250 per plant.
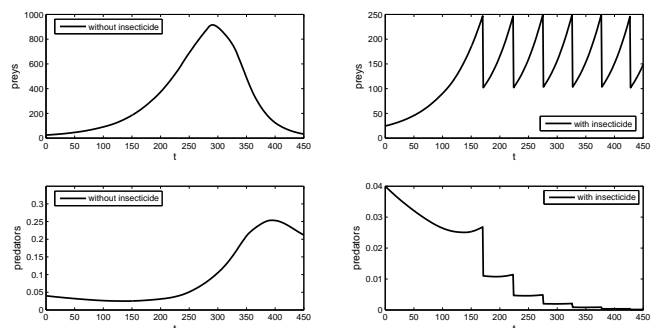

Figure 8: The evolution of the population over time with $x_{0}=24.46$ (initial number of aphids), $y_{0}=0.04$ (initial number of predators) and the temperature from Figure 2 for 2004 and with application of the insecticide.

Figure 8 suggests that the quantity of insecticide was $58.47 \%$ of the quantity recommended by manufacturer and it must be applied in days 26, 31, 35, 39,44 and 48.

Linear regression analysis using the least-squares method was used to estimate the days aforementioned are given by the equation:

$$
d a y=0.084 * t+11.81
$$

where $t$ is the number of iterations given by the computer simulations.

In this case, the quantities of both populations are low small which led to a large increase in the population of the prey and therefore, six low-quantity applications are needed in the period. Besides, the repeated applications, even in low-quantity, are more damaging to predator population than to prey population. 

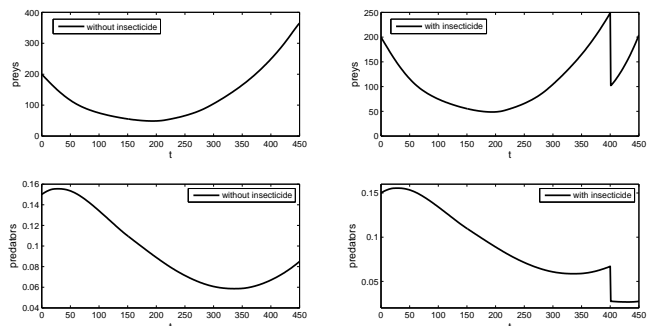

Figure 9: The evolution of the population over time with $x_{0}=500$ (initial number of aphids), $y_{0}=0.15$ (initial number of predators) and the temperature from Figure 2 for 2004 with applications of insecticide.

Figure 9 suggests that the quantity of insecticide was $58.87 \%$ of the quantity recommended by the manufacturer and it must be applied in day 46. The quantity of prey population is low and predators population is large medium and one low-quantity application is enough, because the population of prey excedded 250 per plant (threshold) in the 46th day, demonstrating the importance of the biological control via predation and a constant monitoring of the plantation for the arrival of soybean aphids.

\subsection{Discussion}

The concern about the environment has been increasingly important. Currently, actions aimed at sustainable management of natural resources are goals.

In general, pesticides are toxic, harmful to human health and the environment. One of the most common problems is the contamination of soil, groundwater, rivers and lakes. When the pesticide is used, it intoxicates all life present. Studies show the decrease in the number of pollinating bees and the destruction of bird habitat in environments where pesticides are used [4].

The abusive use of insecticides may lead to an increase number of pests because pests become more resistant, requiring stronger pesticides that will damage the environment even more and will kill the pests' natural predators [4].

The previous affirmations are corroborated in the figures 8 and 9, that is, the use of insecticides in plantations is more damaging to predator population than to prey one. Besides, low-quantities of insecticides from that recommended by the manufacturer may be effective. On one hand, there are the costs for each application. On the other hand, there should be a concern about the environmental damage caused by the abusive use of insecticides in the plantations.

Figure 9 demonstrated the importance of the biological control via predation and a constant monitoring of the plantation from the initial arrival of soybean aphids. Thus, in this paper we propose a planned use of insecticide, based on the amount that harms the insects present in the plant rather than the indiscriminate use.

\section{Conclusions}

This study has suggested that the use of the Mamdani Control Fuzzy in ecology may represent the interaction among species in the environment where the available data are few or qualitative. We have used intuitive hypotheses of the dynamics of aphidsinsidious flower bug and data reported in the literature to elaborate the model without explicit differential equations. It was clear that temperature was an important factor on the growth of aphids population.

We would like to highlight the advantages of using fuzzy rule-based models compared to the deterministic models (differential equations, for example):

- The input and output sets of fuzzy rule-based systems may be easily defined by experts, that is, specialists who may know when the population of a particular species is small, large and so forth of the predators population over time.

- We have used a rule base instead of systems given by equations and this avoid the difficulty of obtaining the parameters.

- If it is necessary to know the parameters, they may be obtained through a curve fitting of the solution generated by the fuzzy model. That is, the parameters may be obtained through a curve fitting procedure from the solution obtained from the fuzzy rule-based model.

- We suggest using this as a policy to control soybean aphids because the fuzzy system provides how often and how much to apply the insecticides on the plants in a simple, intuitive and a direct way.

Future work will develop a simple and specific method using a fuzzy rule-based system to help enhance decision making tools for biological control of this pest.

\section{Acknowledgments}

The first author acknowledges the Coordination for the Improvement of Higher Education Personnel (CAPES) and the second author acknowledges the National Council for Scientific and Technological Development $(\mathrm{CNPq})$, project numbers $305862 / 2013-8$, for the financial support.

\section{References}

[1] L. Edelstein-Keshet. Mathematical Models in Biology, Random House, NY, 1998.

[2] G. J. Klir and B. Yuan. Fuzzy Sets And Fuzzy Logic: Theory and Applications, Prentice Hall, N. Jersey, 1995. 
[3] W. Pedrycs and F. Gomide. An Introduction to Fuzzy Sets: Analysis and Design, Massachusets Institute of Technology, 1998.

[4] D. Pimentel and H. Lchman, editors. The Pesticide question: environment, economics, and ethics, Routledge, Chapman and Hall, New York, 1993.

[5] H. J. S. Yoo and R. J. O'Neil, Temporal relationships between the generalist predator, Orius insidiosus, and its two major prey in soybean, Biological Control, 48:168-180, 2009.

[6] E. H. Mamdani and S. Assilian, An experiment in linguistic synthesis with a fuzzy logic controller, Int. J. Man-Machine Studies, 7:1-13, 1975.

[7] D. W.Ragsdale, D. J. Voegtlin and R. J. O'Neil, Soybean aphid biology in North America, Ann. Entomol. Soc. Am., 97(2):204-208, 2004.

[8] D. W. Ragsdale, B. P. McCornack, R. C. Venette, B. D. Potter, I. V. MacRae, E. W. Hodgson and M.E. O'Neal, Economic Threshold for Soybean Aphid (Hemiptera: Aphididae), Journal of Economic Entomology, 100(4):12581267, 2007.

[9] T. R. Brosius, L. G. Higley, and T. E. Hun, T.E., Population dynamics of soybean aphid and biotic mortality at the edge of its range, Journal of Economic Entomology, 100(4):1268-1275, 2007.

[10] B. P. McCornack, D. W. Ragsdale abd R. C. Venette, Demography of Soybean Aphid (Homoptera: Aphididae) at Summer Temperatures, J. Ec. Entomology, 97(3):854-861, 2004.

[11] M. S. Peixoto, L. C. Barros and R. C. Bassanezi, Predator-prey fuzzy model, Ecological Modelling, 214:39-44, 2008.

[12] L. A. Zadeh, Fuzzy Sets, Informat. Control, $8: 338-353,1965$

[13] T. Hunt, Soybean aphid management in Nebraska. NebFacts. Nebraska Cooperativa Extension. IARN-UNL, Lincoln, NE. (NF04-599), 2005 . 